\title{
Thermoluminescence studies of manganese doped calcium tetraborate (CaB407:Mn) nanocrystal synthesized by co-precipitation method
}

\begin{abstract}
Manganese $(\mathrm{Mn})$ doped bone-equivalent calcium tetraborate $(\mathrm{CaB} 4 \mathrm{O} 7)$ nanocrystals were synthesized using co-precipitation and thermal treatment techniques. The synthesized nanophosphors are found to possess the monoclinic structure and have the particle size about $9 \mathrm{~nm}$. They showed good luminescence quantum efficiency and enough potential for radiation dose measurement in medical and industrial purposes. The variation of dopant concentrations was investigated from 0.1 to $2 \mathrm{~mol} \%$ and it was found that the optimal concentration of $1.4 \mathrm{~mol} \% \mathrm{Mn}$ has the highest sensitivity among other concentrations. The results revealed that $\mathrm{Mn}$ enhance the thermoluminescence (TL) sensitivity about 80 times higher than the un-doped samples at $1 \mathrm{kGy}$ and the TL response over a wide range of doses from $0.05 \mathrm{~Gy}$ to $2.0 \mathrm{kGy}$ increased linearly with increasing the absorbed dose.
\end{abstract}

Keyword: Calcium borate; Nanocrystal; Thermoluminescence; Co-precipitation; Manganese; Radiation dose 